LBNL-51361

MPI-PhT/2002-39

\title{
Non-Abelian Gauge Theory on $q$-Quantum Spaces
}

\author{
S. Schraml \\ Theoretical Physics Group, Bldg. 50A5104 \\ Lawrence Berkeley National Laboratory \\ One Cyclotron Road, Berkeley, CA 94720 \\ and \\ Max-Planck-Institut für Physik \\ Föhringer Ring 6, D-80805 München

\begin{abstract}
Gauge theories on $q$-deformed spaces are constructed using covariant derivatives. For this purpose a "vielbein" is introduced, which transforms under gauge transformations. The non-Abelian case is treated by establishing a connection to gauge theories on commutative spaces, i.e. by a Seiberg-Witten map. As an example we consider the Manin plane. Remarks are made concerning the relation between covariant coordinates and covariant derivatives.
\end{abstract}

e-mail: SLSchraml@lbl.gov 


\section{Introduction}

Gauge theories on noncommutative spaces have recently found some interest in mathematical high energy physics. Most of the work concentrated on noncommutative spaces, for which the commutator of two coordinates is constant: $\left[\hat{x}^{i}, \hat{x}^{j}\right]=i \theta^{i j} \in \mathbb{C}$. From the point of view of gauge theory this space is nice, since the algebra relations of the derivatives do not get deformed (up to possible constants in the commutator of two derivatives). This allows for example to write for the gauge transformation of the components of the gauge potential $\delta A_{i}=\partial_{i} \Lambda+i \Lambda * A_{i}-i A_{i} * \Lambda$; i.e. only the noncommutative $*$-product has to be used instead of the commutative point-wise product. The reason for this is the unchanged Leibniz rule.

However, for more general noncommutative spaces the derivatives - if they exist - and also the Leibniz rule change. A case for which differential calculi have been worked out is the structure of $q$-deformed quantum spaces $[1,2]$. It has the advantage, that not only the space is deformed, but also the symmetries of the space get deformed. In this paper we treat gauge theory on these spaces by introducing covariant derivatives. The case of a general non-Abelian gauge group is dealt with by constructing a Seiberg-Witten map, i.e. a map which connects the gauge theory on the noncommutative space with gauge theory on a commutative space [3]. For this purpose we use the $*$-product formalism; in particular the derivatives become in this formulation power series in the deformation parameter.

We start with recalling some definitions and properties of $q$-deformed spaces $[4,5,6]$, mainly to fix the notation (which we took form [7]). We think of quantum spaces as being defined by the commutation relations of their coordinates $\hat{x}^{1}, \ldots, \hat{x}^{N}$, which are quadratic in our case:

$$
P_{-k l}^{i j} \hat{x}^{k} \hat{x}^{l}=0, \quad P_{-k l}^{i j} \in \mathbb{C} .
$$

This means, that we consider the algebra of formal power series in the elements $\hat{x}^{i}$ modulo the ideal generated by the relations (1):

$$
\mathcal{A}_{\hat{x}} \equiv \frac{\mathbb{C}\left[\left[\hat{x}^{1}, \ldots, \hat{x}^{N}\right]\right]}{P_{-} \hat{x} \hat{x}}
$$

as defining the "noncommutative space". The matrix $P_{-}$is the ( $q$-deformed) anti-symmetric projector appearing in the projector decomposition of the $\hat{R}$ matrix of the respective quantum group:

$$
\begin{array}{lll}
G L_{q}, S L_{q} & : & \hat{R}=q P_{+}-q^{-1} P_{-} \\
S O_{q}, S P_{q} & : & \hat{R}=q P_{+}-q^{-1} P_{-}+q^{1-N} P_{0}
\end{array}
$$

with $\sum P=1$; the $\hat{R}$-matrix fulfills the Yang-Baxter equation:

$$
\hat{R}_{12} \hat{R}_{23} \hat{R}_{12}=\hat{R}_{23} \hat{R}_{12} \hat{R}_{23},
$$


where we used the notation $\hat{R}_{12 j_{1} j_{2} j_{3}}^{i_{1} i_{2} i_{3}} \equiv \hat{R}_{j_{1} j_{2}}^{i_{1} i_{2}} \delta_{j_{3}}^{i_{3}}$ and $\hat{R}_{23 j_{1} j_{2} j_{3}}^{i_{1} i_{2} i_{3}} \equiv \delta_{j_{1}}^{i_{1}} \hat{R}_{j_{2} j_{3}}^{i_{2} i_{3}}$. The parameter $q=e^{h} \in \mathbb{C}$ is the deformation parameter; for $q \rightarrow 1$ one recovers the undeformed commutative space. Given the $\hat{R}$-matrix, the quantum group $G_{q}$ itself is defined by the relations of its (noncommutative) algebra of functions, which is generated by $u_{i}^{j} ; i, j: 1, \ldots, N$ :

$$
\hat{R}_{k l}^{i j} u_{m}^{k} u_{n}^{l}=u_{k}^{i} u_{l}^{j} \hat{R}_{m n}^{k l} .
$$

The noncommutative algebra $\mathcal{A}_{\hat{x}}$ is a comodule algebra of the respective quantum group; and it is a module algebra of the dual of the quantum group, namely of the deformed enveloping algebra $U_{q}(g)$ of the Lie-algebra related to the respective group. The deformed enveloping algebra is generated by the so called $L$-functionals $\hat{\ell}_{i j}^{+}$and $\hat{\ell}_{i j}^{-}, i, j=1 \ldots N$, which satisfy

$$
\hat{R}_{i j}^{l k} \hat{\ell}_{k s}^{ \pm} \hat{\ell}_{l t}^{ \pm}=\hat{\ell}_{j l}^{ \pm} \hat{\ell}_{i k}^{ \pm} \hat{R}_{k l}^{t s}
$$

and for which holds: $\hat{\ell}_{i j}^{+}=0$ for $j<i, \hat{\ell}_{i j}^{-}=0$ for $j>i$.

There exist two well known covariant, w.r.t. the (co-)action of the quantum group, differential calculi for these quantum spaces:

$$
\begin{aligned}
d \hat{x}^{i} d \hat{x}^{j} & =-q^{ \pm 1} \hat{R}_{k l}^{ \pm 1 i j} d \hat{x}^{k} d \hat{x}^{l} \\
\hat{x}^{i} d \hat{x}^{j} & =q^{ \pm 1} \hat{R}_{k l}^{ \pm 1 i j} d \hat{x}^{k} \hat{x}^{l}
\end{aligned}
$$

Here the exponents +1 and -1 correspond to the first and the second differential calculus respectively. The associated derivatives $\hat{\partial}_{i}\left(d=d \hat{x}^{i} \hat{\partial}_{i}\right)$ fulfill

$$
\begin{aligned}
P_{-k l}^{i j} \hat{\partial}_{j} \hat{\partial}_{i} & =0 \\
\hat{\partial}_{i} \hat{x}^{j} & =\delta_{i}^{j}+q^{ \pm 1} \hat{R}^{ \pm 1}{ }_{i k} l \hat{x}^{k} \hat{\partial}_{l}
\end{aligned}
$$

Since in the $q \rightarrow 1$ limit $\hat{R}_{i k}^{j l}$ goes to $\delta_{k}^{j} \delta_{i}^{l}$, this is clearly a deformation of the usual differential calculus. Defining

$$
P_{S} \equiv 1-P_{-}
$$

the commutation relations among the differentials can be written as

$$
P_{S}^{k l} d \hat{x}^{i} d \hat{x}^{j}=0 .
$$

\section{Realization on functions}

In the following we realize the whole noncommutative algebra, including coordinates $\hat{x}$, partial derivatives $\hat{\partial}$ and the deformed enveloping algebra $U_{q}(g)$, as operators acting on the noncommutative coordinate algebra $\mathcal{A}_{\hat{x}}$. I.e. we ask for operators on the algebra $\mathcal{A}_{\hat{x}}$ of coordinates, such that the above relations hold. To distinguish between the elements $\hat{a}$ of the algebra and the corresponding mappings, we will denote the latter by $\check{a}$ :

$$
\check{a}: \mathcal{A}_{\hat{x}} \longrightarrow \mathcal{A}_{\hat{x}} \text {. }
$$


For explicit formulas we use a basis of the algebra $\mathcal{A}_{\hat{x}}$. Due to the YangBaxter equation such a basis is for example given by ordered monomials in the case of $q$-deformed quantum spaces :

$$
\left\{\left(\hat{x}^{1}\right)^{i_{1}}\left(\hat{x}^{2}\right)^{i_{2}} \cdots\left(\hat{x}^{N}\right)^{i_{N}} \mid i_{n}=0,1,2, \ldots\right\} .
$$

Clearly, the coordinates $\hat{x}^{i}$ act on functions by multiplication:

$$
\left(\check{x}^{i} f\right)(\hat{x}) \equiv \hat{x}^{i} f(\hat{x}) .
$$

In terms of a basis this means essentially a reordering.

The quantum group $G_{q}$ coacts on coordinates, which on the other hand also form a vector representation of the deformed enveloping algebra $U_{q}(g)$. Using the $L$-functionals $\hat{\ell}_{i j}^{ \pm}$as generators of $U_{q}(g)$ these operations are connected:

$$
\hat{\ell}_{i j}^{ \pm} \triangleright \hat{x}^{k}=\left\langle\hat{\ell}_{i j}^{ \pm}, u_{k}^{l}\right\rangle \hat{x}^{l}=\hat{R}_{i l}^{ \pm 1 k j} \hat{x}^{l} .
$$

Here we have used the right coaction $\hat{x}^{m} \mapsto \hat{x}^{l} \otimes u_{m}^{l}$, and the dual pairing $\langle\cdot, \cdot\rangle: G_{q} \times U_{q}(g) \rightarrow \mathbb{C}$, which gives rise to a left action $\triangleright$, and which is related to the action according to the vector representation. "Related" means that there might be an additional $q$-factor, if the $R$-matrix is not exactly given by the $R$-matrix $R_{V V}$ of the fundamental vector representation of the $q$-deformed enveloping algebra. This is the case in the example we treat below, in which $R=q^{\frac{1}{2}} R_{V V}$. Since the coproduct of $U_{q}(g)$ is known:

$$
\Delta\left(\hat{\ell}_{i j}^{ \pm}\right)=\hat{\ell}_{i k}^{ \pm} \otimes \hat{\ell}_{k j}^{ \pm},
$$

the action of the operators $\check{\ell}_{i j}^{ \pm}$, i.e. of $U_{q}(g)$, on functions (polynomials in the coordinates $\hat{x}^{i}$ ) is determined.

The commutation relations between the coordinates and the $L$-functionals are obtained by a crossed product construction, that is $a \hat{x}=\sum\left(a_{(1)} \triangleright \hat{x}\right) a_{(2)}$ in our case:

$$
\hat{\ell}_{i k}^{ \pm} \hat{x}^{m}=\hat{R}_{i l}^{ \pm 1 m j} \hat{x}^{l} \hat{\ell}_{j k}^{ \pm}
$$

Here $\hat{R}^{+1}$ appears in the $\hat{\ell}^{+} \hat{x}$-relations and $\hat{R}^{-1}$ in the $\hat{\ell}^{-} \hat{x}$-relations, cf. Eqn. (14). Again, there might be an additional factor in front of $\hat{R}$ according to the relation between $R$ and $R_{V V}$. With this at hand the action on a polynomial in $\hat{x}$ can be obtained as follows: multiply the function from the left with some $\hat{\ell}_{i j}^{ \pm}$, commute this $\hat{\ell}_{i j}^{ \pm}$and all $\hat{\ell}^{ \pm}$obtained during this process to the whole right, set all $\hat{\ell}_{k k}^{ \pm}$equal to 1 and skip all terms with a $\hat{\ell}_{k l}^{ \pm}, k \neq l$ on the right.

For the partial derivatives we first determine the commutation relations between differentials $d \hat{x}^{i}$ and functions $f(\hat{x})$. We treat the first differential calculus and start with relation (7):

$$
\begin{aligned}
\hat{x}^{i} d \hat{x}^{j} & =q \hat{R}_{k l}^{i j} d \hat{x}^{k} \hat{x}^{l} \\
& =q\left\langle\hat{\ell}_{k j}^{+}, u_{i}^{l}\right\rangle \hat{x}^{k} \hat{x}^{l} \\
& =q d \hat{x}^{k} \hat{\ell}_{k j}^{+} \triangleright \hat{x}^{i} .
\end{aligned}
$$


To keep track of the $q$ on the right hand side we extend our algebra by a scaling operator ${ }^{1} \hat{U}$ for which holds:

$$
\hat{U} \hat{x}^{i}=q \hat{x}^{i} \hat{U}, \quad \hat{U} \hat{\partial}_{i}=q^{-1} \hat{\partial}_{i} \hat{U}, \quad \hat{U} \hat{\ell}_{i j}^{ \pm}=\hat{\ell}_{i j}^{ \pm} \hat{U} .
$$

On functions the scaling operator is realized by

$$
\begin{aligned}
\check{U} f(\hat{x}) & =f(q \hat{x}) \\
\check{U}(f g) & =(\check{U} f)(\check{U} g) .
\end{aligned}
$$

If we take into account the coproduct (15) of the $L$-functionals, or in other words the quasitriangularity of the deformed enveloping algebra, we arrive at

$$
f(\hat{x}) d \hat{x}^{j}=d \hat{x}^{k}\left(\hat{U} \hat{\ell}_{k j}^{+}\right) \triangleright f(\hat{x}) \equiv d \hat{x}^{k}\left(B_{k}^{j} f(\hat{x})\right) .
$$

Where we defined the operator

$$
B_{i}{ }^{j}=\check{U} \check{\ell}_{i j}^{+}
$$

which fulfills

$$
B_{i}{ }^{j}(f g)=\left(B_{i}{ }^{k} f\right)\left(B_{k}{ }^{j} g\right)
$$

and

$$
\hat{R}_{i j}^{l k} B_{k}{ }^{s} B_{l}{ }^{t}=B_{j}{ }^{l} B_{i}{ }^{k} \hat{R}_{k l}^{t s}
$$

due to the same relations for the $L$-functionals. This means, that the $B_{i}{ }^{j}$ form a realization of the quantum group.

If we had started with the second differential calculus, we would have obtained $B_{i}{ }^{j}=\check{U}^{-1} \check{\ell}_{i j}^{-}$, where we extended the algebra by the inverse of $U$.

Applying $d=d \hat{x}^{i} \hat{\partial}_{i}$ to a function we define the realization $\check{\partial}_{i}$ of the partial derivatives on functions $f$ as follows:

$$
d f=d \hat{x}^{i}\left[\left(\check{\partial}_{i} f\right)+\left(B_{i}^{j} f\right) \hat{\partial}_{j}\right] .
$$

We obtain therefore the Leibniz rule

$$
\check{\partial}_{i}(f g)=\left(\check{\partial}_{i} f\right) g+\left(B_{i}{ }^{j} f\right)\left(\check{\partial}_{j} g\right) .
$$

Eqn. (22) ensures that $\check{\partial}_{i}(f(g h))=\check{\partial}_{i}((f g) h)$, which must hold since the algebra $\mathcal{A}_{\hat{x}}$ of coordinates is associative.

Eqn. (24) shows, that the explicit formulas for the action of the derivatives $\check{\partial}_{i}$ on functions $f(\hat{x})$ can be obtained in the same way as described above for the $L$-functionals. Namely, commute $\hat{\partial}_{i}$ from the left side to the right side of $f(\hat{x})$ and forget about terms with some derivative $\hat{\partial}$ on the right.

\footnotetext{
${ }^{1}$ It corresponds to the scaling operator which one uses to construct real momentum operators $[8,9]$.
} 
Since the differential calculus is covariant w.r.t. the quantum group, we obtain for the commutation relation between the $L$-functionals and the derivatives:

$$
\hat{\partial}_{m} \hat{\ell}_{l j}^{ \pm}=\hat{R}_{l m}^{ \pm 1 i k} \hat{\ell}_{k j}^{ \pm} \hat{\partial}_{i}
$$

As above for the coordinates, we have $\hat{\partial} \hat{\ell}^{+}=\hat{R}^{+1} \hat{\ell}^{+} \hat{\partial}$ and $\hat{\partial} \hat{\ell}^{-}=\hat{R}^{-1} \hat{\ell}^{-} \hat{\partial}$ respectively. Using Eqn. (21) we therefore obtain:

$$
\check{\partial}_{m} B_{l}^{j}=q \hat{R}_{l m}^{i k} B_{k}{ }^{j} \check{\partial}_{i} .
$$

The action on products of functions (25) can be interpreted as comultiplication:

$$
\begin{aligned}
\Delta\left(\partial_{i}\right) & =\partial_{i} \otimes 1+B_{i}{ }^{j} \otimes \partial_{j} \\
\Delta(U) & =U \otimes U
\end{aligned}
$$

One can show, that this together with (15) and the counit

$$
\varepsilon\left(\ell_{i j}^{ \pm}\right)=\delta_{i j}, \quad \varepsilon\left(\partial_{i}\right)=0, \quad \varepsilon\left(U^{ \pm 1}\right)=1
$$

defines a bialgebra structure on the algebra generated by $\ell_{i j}^{ \pm}, \partial_{i}$ and $U^{ \pm 1}$. Since the action of the antipode of $U_{q}(g)$ on the $L$-functionals is known, we can equip this bialgebra easily with a antipode $S$ to get a Hopf algebra:

$$
S\left(U^{ \pm 1}\right)=U^{\mp 1}, \quad S\left(\partial_{i}\right)=-S\left(B_{i}{ }^{k}\right) \partial_{k} .
$$

Indeed, in this way we get two bialgebra structures, since we have either $B_{i}{ }^{j}=$ $U \ell_{i j}^{+}$or $B_{i}{ }^{j}=U^{-1} \ell_{i j}^{-}$, depending on which differential calculus we use. The relations among $\ell_{i j}^{ \pm}, \partial_{i}$ and $U^{ \pm 1}$ are the same in both cases. However, the algebra $\mathcal{A}_{\hat{x}}$ of coordinates, on which this algebra acts in two different ways, is only a module algebra of this bialgebra if one chooses the appropriate comultiplication.

Below we will connect the gauge theory on the noncommutative space with gauge theory on commutative spaces. This will be done using *-products [10]. For the construction of a $*$-product resembling a given algebra $\mathcal{A}_{\hat{x}}$ we use the basis for which we have obtained the action of all operators above. To be specific we take the basis (12), i.e. normal ordering. We define a map $W$ ("quantization") from the algebra of $N$ commuting variables $x^{1}, \ldots, x^{N}$ to $\mathcal{A}_{\hat{x}}$ by

$$
W\left(\left(x^{1}\right)^{i_{1}} \cdots\left(x^{N}\right)^{i_{N}}\right) \equiv\left(\hat{x}^{1}\right)^{i_{1}} \cdots\left(\hat{x}^{N}\right)^{i_{N}} .
$$

The requirement, that this map is an algebra homomorphism defines a $*$-product for polynomials of commuting variables:

$$
W(f * g) \equiv W(f) W(g) .
$$

Since in the case of quantum spaces $W$ is clearly invertible (due to the PoincaréBirkhoff-Witt property), the $*$-product exists. This $*$-product can be written in 
terms of a formal power series of differential operators with formal parameter $h \equiv \ln q$. Formally we can extend it to $C^{\infty}\left(\mathbb{R}^{N}\right)[[h]]$.

Furthermore we are able to translate not only the product of functions, but also operators acting on the noncommutative algebra $\mathcal{A}_{\hat{x}}$. Given some operator $\check{O}$ on $\mathcal{A}_{\hat{x}}$ this is done by

$$
W(\check{O} f) \equiv \check{O} W(f)
$$

Therefore we get for all the operators $\check{\ell}_{i j}^{ \pm}, \check{\partial}_{i}, \check{U}^{ \pm 1}$ defined above, corresponding differential operators acting on $C^{\infty}$-functions on $\mathbb{R}^{N}$. These operators are power series in the deformation parameter (and fulfill by definition the algebra relations of $\left.\ell_{i j}^{ \pm}, \partial_{i}, U^{ \pm 1}\right)$.

As an illustration we consider the $q$-deformed two-dimensional plane (Maninplane), which has a $S L_{q}(2)$-symmetry. For more complicated examples see [11]. The well known $\hat{R}$-matrix is given by

$$
\hat{R}=\left(\begin{array}{cccc}
q & 0 & 0 & 0 \\
0 & \lambda & 1 & 0 \\
0 & 1 & 0 & 0 \\
0 & 0 & 0 & q
\end{array}\right)
$$

where $\lambda=q-q^{-1}$. If we consider the first differential calculus $\left(\hat{x}^{i} d \hat{x}^{j}=\right.$ $q \hat{R}_{k l}^{i j} d \hat{x}^{k} \hat{x}^{l}$ ) we obtain the following relations:

$$
\begin{aligned}
& \hat{x}^{1} \hat{x}^{2}=q \hat{x}^{2} \hat{x}^{1} \\
& \hat{\partial}_{1} \hat{\partial}_{2}=q^{-1} \hat{\partial}_{2} \hat{\partial}_{1} \\
& \hat{\partial}_{1} \hat{x}^{1}=1+q^{2} \hat{x}^{1} \hat{\partial}_{1}+q \lambda \hat{x}^{2} \hat{\partial}_{2}, \quad \hat{\partial}_{1} \hat{x}^{2}=q \hat{x}^{2} \hat{\partial}_{1} \\
& \hat{\partial}_{2} \hat{x}^{1}=q \hat{x}^{1} \hat{\partial}_{2}, \quad \hat{\partial}_{2} \hat{x}^{2}=1+q^{2} \hat{x}^{2} \hat{\partial}_{2}
\end{aligned}
$$

For the relations between the coordinates and the $U_{q}\left(s l_{2}\right)$-generators one has to take into account a $q^{\frac{1}{2}}$ due to the relation between the $R$-matrix (34) and the $R$-matrix for the vector representation $R_{V V}$. This implies $\hat{U} \hat{x}^{i}=q^{\frac{3}{2}} \hat{x}^{i} \hat{U}$; using the second differential calculus would however require a scaling by $q^{-\frac{1}{2}}$. We obtain $\left(\ell_{21}^{+}=0\right)$ :

$$
\begin{aligned}
& \hat{\ell}_{11}^{+} \hat{x}^{1}=q^{\frac{1}{2}} \hat{x}^{1} \hat{\ell}_{11}^{+}, \quad \hat{\ell}_{12}^{+} \hat{x}^{1}=q^{-\frac{1}{2}}\left(q \hat{x}^{1} \hat{\ell}_{12}^{+}+\lambda \hat{x}^{2} \hat{\ell}_{22}^{+}\right), \quad \hat{\ell}_{22}^{+} \hat{x}^{1}=q^{-\frac{1}{2}} \hat{x}^{1} \hat{\ell}_{22}^{+} \\
& \hat{\ell}_{11}^{+} \hat{x}^{2}=q^{-\frac{1}{2}} \hat{x}^{2} \hat{\ell}_{11}^{+}, \quad \hat{\ell}_{12}^{+} \hat{x}^{2}=q^{-\frac{1}{2}} \hat{x}^{2} \hat{\ell}_{12}^{+}, \quad \hat{\ell}_{22}^{+} \hat{x}^{2}=q^{\frac{1}{2}} \hat{x}^{2} \hat{\ell}_{22}^{+}
\end{aligned}
$$

For the explicit realization on functions we take the basis $\left(\hat{x}^{1}\right)^{n}\left(\hat{x}^{2}\right)^{m}$. The, 
in this case simple, calculations yield:

$$
\begin{aligned}
\check{\partial}_{1}\left(\left(\hat{x}^{1}\right)^{n}\left(\hat{x}^{2}\right)^{m}\right) & =q^{n+2 m-1}[n]\left(\hat{x}^{1}\right)^{n-1}\left(\hat{x}^{2}\right)^{m} \\
\check{\partial}_{2}\left(\left(\hat{x}^{1}\right)^{n}\left(\hat{x}^{2}\right)^{m}\right) & =q^{n+m-1}[m]\left(\hat{x}^{1}\right)^{n}\left(\hat{x}^{2}\right)^{m-1} \\
\check{\ell}_{11}^{+}\left(\left(\hat{x}^{1}\right)^{n}\left(\hat{x}^{2}\right)^{m}\right) & =q^{\frac{1}{2}(n-m)}\left(\hat{x}^{1}\right)^{n}\left(\hat{x}^{2}\right)^{m} \\
\check{\ell}_{12}^{+}\left(\left(\hat{x}^{1}\right)^{n}\left(\hat{x}^{2}\right)^{m}\right) & =\lambda q^{\frac{1}{2}(m-n)}[n]\left(\hat{x}^{1}\right)^{(n-1)}\left(\hat{x}^{2}\right)^{(m+1)} \\
\check{\ell}_{22}^{+}\left(\left(\hat{x}^{1}\right)^{n}\left(\hat{x}^{2}\right)^{m}\right) & =q^{-\frac{1}{2}(n-m)}\left(\hat{x}^{1}\right)^{n}\left(\hat{x}^{2}\right)^{m} \\
\check{\ell}_{21}^{-}\left(\left(\hat{x}^{1}\right)^{n}\left(\hat{x}^{2}\right)^{m}\right) & =-\lambda q^{\frac{1}{2}(n-m)+1}[m]\left(\hat{x}^{1}\right)^{(n+1)}\left(\hat{x}^{2}\right)^{(m-1)} \\
\check{U}\left(\left(\hat{x}^{1}\right)^{n}\left(\hat{x}^{2}\right)^{m}\right) & =q^{\frac{3}{2}(n+m)}\left(\hat{x}^{1}\right)^{n}\left(\hat{x}^{2}\right)^{m}
\end{aligned}
$$

In these equations $\lambda=q-q^{-1}$ and $[n]=\frac{q^{n}-q^{-n}}{q-q^{-1}}$ are defined as usual.

Using the basis $\left(\hat{x}^{1}\right)^{n}\left(\hat{x}^{2}\right)^{m}, n, m \in \mathbb{N}$, the $*$-product is found to be [12]

$$
f * g=\left.q^{-y^{1} \frac{\partial}{\partial y^{1}} x^{2} \frac{\partial}{\partial x^{2}}} f\left(x^{1}, x^{2}\right) g\left(y^{1}, y^{2}\right)\right|_{\substack{y^{1} \rightarrow x^{1} \\ y^{2} \rightarrow x^{2}}}
$$

In the case of the higher-dimensional $G L_{q^{-}}$and $S L_{q^{-}}$-quantum planes the *product is quite simple and very similar to the 2-dimensional. For Euclidian quantum spaces however the formulas become complicated [13].

The derivatives $\check{\partial}_{i}$ and the $B_{i}{ }^{j}$ operators become $\left(\partial_{i}=\frac{\partial}{\partial x^{i}}\right)$ :

$$
\begin{aligned}
\check{\partial}_{1} f & =\frac{1}{\lambda x^{1}} q^{2 x^{2} \partial_{2}-1}\left(q^{2 x^{1} \partial_{1}}-1\right) f \\
\check{\partial}_{2} f & =\frac{1}{\lambda x^{2}} q^{x^{1} \partial_{1}-1}\left(q^{2 x^{2} \partial_{2}}-1\right) f \\
\check{B}_{1}{ }^{1} f & =q^{2 x^{1} \partial_{1}+x^{2} \partial_{2} f} \\
\check{B}_{1}{ }^{2} f & =\frac{x^{2}}{x^{1}} q^{2 x^{2} \partial_{2}}\left(q^{2 x^{1} \partial_{1}}-1\right) f \\
\check{B}_{2}{ }^{2} f & =q^{x^{1} \partial_{1}+2 x^{2} \partial_{2}} f
\end{aligned}
$$

Using $\frac{1}{x} f\left(x \partial_{x}\right)=f\left(x \partial_{x}+1\right) \frac{1}{x}$, it is easy to check explicitly, that the algebra relations are really satisfied. I.e. the algebra is realized by differential operators acting on $C^{\infty}$-functions on $\mathbb{R}^{N}$.

\section{Covariant derivatives}

In the following we treat gauge theory on quantum spaces by constructing covariant derivatives.

We assume that an infinitesimal gauge transformations of a field $\psi(\hat{x})$, which belongs to a representation of the gauge group, is given by:

$$
\delta \psi(\hat{x})=i \Lambda(\hat{x}) \psi(\hat{x}) .
$$


One might think of the gauge parameter $\Lambda(\hat{x})$ as a matrix and of $\psi(\hat{x})$ as a column vector, both with entries in $\mathcal{A}_{\hat{x}}$; see below, section 4 .

We require $\delta\left(\mathcal{D}_{i} \psi\right)=i \Lambda\left(\mathcal{D}_{i} \psi\right)$ for the covariant derivation of a field and make for this purpose the ansatz:

$$
\mathcal{D}_{i} \psi \equiv E_{i}{ }^{j}\left(\check{\partial}_{j}-i A_{j}\right) \psi .
$$

Where we introduced a gauge field $A_{i}$ and a "vielbein" field $E_{i}{ }^{j}$ which we assume to be invertible:

$$
E_{i}{ }^{k} e_{k}^{j}=\delta_{i}^{j}=e_{i}^{k} E_{k}^{j}
$$

From the requirement of covariance under gauge transformations we obtain:

$$
\begin{aligned}
& \delta E_{i}{ }^{j}=i \Lambda E_{i}{ }^{j}-i E_{i}{ }^{k}\left(B_{k}{ }^{j} \Lambda\right) \\
& \delta A_{i}=\check{\partial}_{i} \Lambda+i\left(B_{i}{ }^{j} \Lambda\right) A_{j}-i A_{i} \Lambda
\end{aligned}
$$

The inverse of the vielbein transforms according to

$$
\delta e_{i}{ }^{j}=i\left(B_{i}{ }^{k} \Lambda\right) e_{k}{ }^{j}-i e_{i}{ }^{j} \Lambda .
$$

For $j<i$ the operators $B_{i}{ }^{j}$ vanish for the above choice (21). Therefore it is compatible with the transformation law (43) to choose $E_{i}{ }^{j} \equiv 0 \equiv e_{i}{ }^{j}$ for $j<i$. With this choice it is easy to express $e_{i}{ }^{j}$ in terms of $E_{i}{ }^{j}$.

To obtain tensors we apply two covariant derivatives to a field. Using the definition (41) and the relation (8) for the partial derivatives, we arrive at

$$
\mathcal{D}_{i} \mathcal{D}_{j} \psi=G_{i j}{ }^{k l} \mathcal{D}_{k} \mathcal{D}_{l} \psi+T_{i j}{ }^{k} \mathcal{D}_{k} \psi+F_{i j} \psi
$$

Where $G_{i j}{ }^{k l}$, the torsion $T_{i j}{ }^{k}$ and the field strength $F_{i j}$ transform as tensors:

$$
\delta G_{i j}{ }^{k l}=i\left[\Lambda, G_{i j}{ }^{k l}\right], \quad \delta T_{i j}{ }^{k}=i\left[\Lambda, T_{i j}{ }^{k}\right], \quad \delta F_{i j}=i\left[\Lambda, F_{i j}\right] .
$$

Explicitly one finds the following expressions for these tensors:

$$
\begin{aligned}
G_{i j}{ }^{k l} & =E_{i}{ }^{m}\left(B_{m}{ }^{r} E_{j}{ }^{n}\right) P_{S n r}{ }^{t s}\left(B_{s}{ }^{u} e_{t}{ }^{l}\right) e_{u}{ }^{k} \\
T_{i j}{ }^{k}= & {\left[\left(\mathcal{D}_{i} E_{j}{ }^{m}\right)-G_{i j}{ }^{r n}\left(\mathcal{D}_{r} E_{n}{ }^{m}\right)\right] e_{m}{ }^{k} } \\
i F_{i j}= & E_{i}{ }^{k}\left(B_{k}{ }^{r} E_{j}{ }^{n}\right)\left[\left(\check{\partial ̆}_{r} A_{n}\right)+i\left(B_{r}{ }^{u} A_{n}\right) A_{u}\right. \\
- & \left.-P_{S n r}{ }^{t s}\left(\left(\check{\partial}_{s} A_{t}\right)+i\left(B_{s}{ }^{u} A_{t}\right) A_{u}\right)\right] \\
= & E_{i}{ }^{k}\left(B_{k}{ }^{r} E_{j}{ }^{n}\right) P_{-n r}^{t s}\left(\check{\partial}_{s} A_{t}+i\left(B_{s}{ }^{u} A_{t}\right) A_{u}\right)
\end{aligned}
$$

The covariant derivative $\mathcal{D}_{i} E_{j}{ }^{k}$ of the vielbein, which appears in the expression for the torsion $T_{i j}{ }^{k}$, turns out to be

$$
\mathcal{D}_{i} E_{j}{ }^{k}=E_{i}{ }^{m}\left[\left(\check{\partial}_{m}-i A_{m}\right) E_{j}{ }^{k}+i q\left(B_{m}{ }^{r} E_{j}{ }^{n}\right) \hat{R}_{n r}{ }^{t s}\left(B_{s}{ }^{k} A_{t}\right)\right] .
$$

The zeroth order contribution in the expansion of $F_{i j}$ in powers of $h$ clearly reduces to its usual commutative counterpart, at least up to a normalization. 
We assume that the field strength is anti-symmetric, where anti-symmetric has to be understood in a $q$-deformed sense:

$$
P_{-j i}^{r n} F_{n r}=F_{i j}
$$

Then, inspecting the explicit expression for the field strength (47), one finds that this requirement is fulfilled, if the following relation for the vielbein field holds:

$$
E_{i}^{k}\left(B_{k}^{r} E_{j}^{n}\right) P_{-}^{t s}=P_{-j i}^{r n} E_{n}^{k}\left(B_{k}^{s} E_{r}^{t}\right) .
$$

This condition is covariant under gauge transformations. Since $P_{S}=1-P_{-}$, it holds in the same way for $P_{S}$. If it holds the expressions for the tensors $G_{i j}{ }^{k l}$ and $T_{i j}{ }^{k}$ simplify as follows:

$$
\begin{aligned}
G_{i j}{ }^{k l} & =\left(1-P_{-}\right)_{j i}^{l k} \\
T_{i j}{ }^{k} & =P_{-j i}^{n r}\left(\mathcal{D}_{r} E_{n}{ }^{m}\right) e_{m}{ }^{k},
\end{aligned}
$$

which leads to

$$
P_{-k l}^{i j} \mathcal{D}_{j} \mathcal{D}_{i}=T_{l k}{ }^{m} \mathcal{D}_{m}+F_{l k}
$$

If we define

$$
e^{i} \equiv d \hat{x}^{j} e_{j}^{i}
$$

the condition (50) implies on the level of differentials

$$
P_{S i j}^{k l} e^{i} e^{j}=0 .
$$

It is also possible to consider finite gauge transformations:

$$
\psi(\hat{x}) \mapsto \psi^{\prime}(\hat{x})=G(\hat{x}) \psi(\hat{x}) .
$$

The related transformations of the gauge and vielbein fields are

$$
\begin{aligned}
E_{i}^{\prime j} & =G E_{i}{ }^{k}\left(B_{k}{ }^{j} G^{-1}\right) \\
A_{i}^{\prime} & =-i\left(\check{\partial}_{i} G\right) G^{-1}+\left(B_{i}{ }^{j} G\right) A_{j} G^{-1} .
\end{aligned}
$$

Clearly, this reduces to Eqn. (43) for $G=1+i \Lambda$ with $\Lambda$ infinitesimal small. Accordingly pure gauge fields are given by

$$
\begin{aligned}
E_{i}{ }^{j} & =G\left(B_{i}{ }^{j} G^{-1}\right) \\
A_{i} & =-i\left(\check{\partial}_{i} G\right) G^{-1}=i\left(B_{i}{ }^{j} G\right)\left(\check{\partial}_{j} G^{-1}\right) .
\end{aligned}
$$

For these fields the field strength $F_{i j}$ and the torsion $T_{i j}{ }^{k}$ vanish. Also the relation (50) is satisfied. 


\section{Seiberg-Witten map}

In this section we connect, for an arbitrary non-Abelian gauge group, gauge theories on noncommutative spaces with gauge theories on commutative spaces [3]. This means, that we will express the transformation parameter $\Lambda$, the gauge field $A_{i}$ and the vielbein $E_{i}{ }^{j}$ in terms of the commutative gauge parameter and field $a_{i}$. We follow the cohomological approach of $[14,15]$. For this purpose we introduce a ghost field which is a Lie algebra-valued Grassmannian field: $c=c_{a} T^{a},\left\{c_{a}, c_{b}\right\}=0,\left[T^{a}, T^{b}\right]=i f^{a b}{ }_{c} T^{c}$. As usual the BRST transformations of the respective fields read:

$$
\begin{aligned}
s c & =i c^{2} \\
s a_{i} & =\partial_{i} c+i\left[c, a_{i}\right] \\
s \psi & =i c \psi .
\end{aligned}
$$

Where $\partial_{i}$ is the usual derivative of commuting variables. The BRST operator $s$ is nilpotent $s^{2}=0$ and commutes with the $*$-product, the quantum derivatives $\check{\partial}_{i}$ and the operators $B_{i}{ }^{j}$.

The noncommutative gauge parameter $\Lambda$ is replaced by a noncommutative ghost field $C$, which becomes a functional of the commutative gauge field $a_{i}$ and ghost field $c$. The relation to be fulfilled by $C$ is

$$
s C=i C * C
$$

Once this is solved for $C$, one uses the Eqns:

$$
\begin{aligned}
s E_{i}{ }^{j} & =i C * E_{i}{ }^{j}-i E_{i}{ }^{k} *\left(B_{k}{ }^{j} C\right) \\
s A_{i} & =\check{\partial}_{i} C+i\left(B_{i}{ }^{j} C\right) * A_{j}-i A_{i} * C \\
s \psi & =i C * \psi
\end{aligned}
$$

to determine $E_{i}{ }^{j}, A_{i}$ and $\psi$. It is easily seen, that these relations are consistent with the nilpotency of the BRST operator $s$. The fields $C, A_{i}, E_{i}{ }^{j}$ are formal power series in the deformation parameter $h \equiv \ln q$ :

$$
C=\sum_{n=0}^{\infty} h^{n} C^{(n)}\left[c, a_{l}\right], \quad A_{i}=\sum_{n=0}^{\infty} h^{n} A_{i}^{(n)}\left[a_{l}\right], \quad E_{i}{ }^{j}=\sum_{n=0}^{\infty} h^{n} E^{(n)}{ }_{i}^{j}\left[a_{l}\right],
$$

where each $C^{(n)}\left[c, a_{l}\right]$ is a local functional of $c$ and $a_{l}$; the gauge potential $A_{i}^{(n)}\left[a_{l}\right]$ and the vielbein $E^{(n)}{ }_{i}{ }^{j}\left[a_{l}\right]$ are local functionals of the commutative gauge field $a_{l}$. Furthermore we require for the zeroth order contributions

$$
C^{(0)}=c, \quad A^{(0)}{ }_{i}=a_{i}, \quad E^{(0)}{ }_{i}^{j}=\delta_{i}^{j},
$$

which implies, that we obtain the usual commutative gauge theory in the limit $h \rightarrow 0$.

Solving Eqns. (59) and (60) one finds, that the ghost field $C$, the gauge field $A_{i}$ and the vielbein $E_{i}{ }^{j}$ have to take values in the enveloping algebra of the Lie-algebra of the gauge group [16]. 
It turns out, that at each level the Eqns. (59) and (60) can be written in the form (e.g. for the ghost field) ${ }^{2}$

$$
\Delta C^{(m)}=Z^{(m)}, \quad \Delta \cdot \equiv s \cdot-i[c, \cdot]
$$

with $[\cdot, \cdot]$ being the graded commutator. $Z^{(m)}$ depends on the lower order terms $C^{(n)}, n<m$ (as do $A^{(n)}{ }_{i}$ and $E^{(n)}{ }_{i}{ }^{j}$ respectively) only. The operator $\Delta$ is a graded derivation, its square is zero:

$$
\Delta^{2}=0
$$

Therefore for Eqn. (63) to possess a solution the following consistency relation must hold:

$$
\Delta Z^{(m)}=0
$$

This is indeed the case, as was shown for the ghost field in [17]. By a similar calculation this can also be shown for $A_{i}$ and $E_{i}{ }^{j}$, which should fulfill (60). However, one now has to take into account, that the operators $\check{\partial}_{i}$ and $B_{i}{ }^{j}$ are power series in the deformation parameter $h$.

The existence of the Seiberg-Witten map depends therefore on the triviality of the respective cohomology classes of the coboundary operator $\Delta$. Since in [14] a homotopy operator $(K, K \Delta+\Delta K=1)$ was constructed, this is indeed the case.

The cohomological approach makes it clear, that there is at each order some freedom in the solution of the Eqns. (59), (60); namely, if $C^{(m)}$ is a solution of (63), then $\tilde{C}^{(m)}=C^{(m)}+\Delta H$, with $H$ an arbitrary expression of the gauge field $a_{i}$, is also a solution.

For the example of the 2-dimensional quantum plane we obtain the following solutions to first nontrivial order [18]. The noncommutative gauge parameter has to fulfill

$$
\Delta C^{(1)}=-i x^{1} x^{2} \partial_{2} c \partial_{1} c
$$

which has as solution

$$
C=c+h \frac{i}{2} x^{1} x^{2}\left[\left(\partial_{2} c\right) a_{1}-a_{2}\left(\partial_{1} c\right)\right]+\mathcal{O}\left(h^{2}\right) .
$$

In the case of the vielbein and the gauge potential one has to expand the derivates $\check{\partial}_{i}$ and the operators $B_{i}{ }^{j}$ using the expressions (39) as a power series in $h$. E.g. $E^{(1)}{ }_{1}^{1}$ must fulfill

$$
\Delta E^{(1)}{ }_{1}^{1}=-i\left(2 x^{1} \partial_{1} c+x^{2} \partial_{2} c\right) .
$$

${ }^{2}$ Notice, that this $\Delta$ is different from the $\Delta$ (coproduct) used in section 2. There should be no confusion, since the context is different. 
Which is easily solved:

$$
\begin{aligned}
& E_{1}{ }^{1}=1-i h\left(2 x^{1} a_{1}+x^{2} a_{2}\right)+\mathcal{O}\left(h^{2}\right) \\
& E_{1}{ }^{2}=-i h 2 x^{2} a_{1}+\mathcal{O}\left(h^{2}\right) \\
& E_{2}{ }^{1}=0 \\
& {E_{2}}^{2}=1-i h\left(x^{1} a_{1}+2 x^{2} a_{2}\right)+\mathcal{O}\left(h^{2}\right)
\end{aligned}
$$

Using the ansatz

$$
A_{i}=a_{i}+h A_{i}^{(1)}+\mathcal{O}\left(h^{2}\right)
$$

and the first order solution (67) of the ghost field $C$, one obtains as possible solution to the second of the Eqns. (60):

$$
\begin{aligned}
A_{1}^{(1)}= & \left(2 x^{2} \partial_{2}+x^{1} \partial_{1}\right) a_{1}+i 2 x^{2} a_{1} a_{2}-\frac{i}{2} x^{2} a_{2} a_{1}+i x^{1} a_{1} a_{1} \\
& +\frac{i}{2} x^{1} x^{2}\left[-f_{12} a_{1}+\partial_{2} a_{1} a_{1}-a_{2} \partial_{1} a_{1}\right] \\
A_{2}^{(1)}= & \left(x^{1} \partial_{1}+x^{2} \partial_{2}\right) a_{2}+\frac{i}{2} x^{1} a_{2} a_{1}+i x^{2} a_{2} a_{2} \\
& +\frac{i}{2} x^{1} x^{2}\left[-a_{2} f_{12}-a_{2} \partial_{1} a_{2}+\partial_{2} a_{2} a_{1}\right] .
\end{aligned}
$$

Where we used the commutative field strength $f_{12}=\partial_{1} a_{2}-\partial_{2} a_{1}-i\left[a_{1}, a_{2}\right]$.

\section{Derivatives vs. Coordinates}

For a general noncommutative space it is possible to formulate gauge theory by using covariant coordinates $[12,19]$. Therefore one might ask, if there is a connection between the formulations using derivatives and coordinates respectively.

For example, in the case of the canonical noncommutative structure

$$
\left[\hat{x}^{i}, \hat{x}^{j}\right]=i \theta^{i j}, \quad \theta^{i j} \in \mathbb{C}
$$

it is easily seen, that, for $\theta^{i j}$ invertible, the identification

$$
\hat{\partial}_{i}=-i \theta_{i j} \hat{x}^{j}, \quad \text { with } \theta_{i j}=\left(\theta^{-1}\right)_{i j}
$$

is algebraical consistent, if one allows for noncommuting derivatives $\left[\hat{\partial}_{i}, \hat{\partial}_{j}\right]=$ $i \theta_{i j}$. The respective gauge fields are then simply related by

$$
A_{\text {coord }}^{i}=i \theta^{i j} A_{\text {deriv }, j} .
$$

At least for some $q$-deformed quantum spaces such relations between derivatives and coordinates also exist; but they are more complicated. These relations 
include in general also the scaling operator $U$ and the generators $\ell_{i j}^{ \pm}$of the deformed enveloping algebra $U_{q}(g)$, and furthermore the algebra must be extended by the inverses of certain of its elements.

For $q$-deformed Euclidean spaces (i.e. spaces covariant under $S O_{q}$ ) it is possible to express the differential $d f$ of a function as a commutator [20]:

$$
(1-q) d f=[\omega, f] \equiv \frac{q^{2}}{1+q^{2}}\left[\hat{r}^{-2} d\left(\hat{r}^{2}\right), f\right] .
$$

Where $\hat{r}^{2}=g_{i j} \hat{x}^{i} \hat{x}^{j}$ is the $q$-deformed length, which is central in the algebra of the coordinates and (co-)invariant under the action of the quantum group. Its differential can be written as $d\left(\hat{r}^{2}\right)=D_{k l} d \hat{x}^{k} \hat{x}^{l}$, with $D_{k l}=\left(1+q^{-1}\right) g_{k l}$. Now, assuming that there are some operators $C_{i}^{l}$ such that $f(\hat{x}) \hat{x}^{l} \equiv \hat{x}^{i} C_{i}^{l} f(\hat{x})$, we obtain

$$
[\omega, f]=\frac{1}{1+q} d \hat{x}^{k}\left[D_{k l} \frac{\hat{x}^{l}}{\hat{r}^{2}}-\frac{1}{\hat{r}^{2}} D_{m l} \hat{x}^{i} C_{i}^{l} B_{k}{ }^{m}\right] f
$$

which implies

$$
\hat{\partial}_{k}=\frac{1}{1-q^{2}} \frac{1}{\hat{r}^{2}}\left(D_{k l} \hat{x}^{l}-D_{m l} \hat{x}^{i} C_{i}^{l} B_{k}^{m}\right) .
$$

Even for our example, the Manin plane, which is not Euclidean, one can read off the following identifications from Eqns. (37):

$$
\begin{aligned}
& \check{\partial}_{1}=+\lambda^{-1} \check{V}^{2} \check{\ell}_{11}^{-} \check{\ell}_{12}^{+} \frac{1}{\check{x}^{2}} \\
& \check{\partial}_{2}=-\lambda^{-1} \check{V}^{2} \check{\ell}_{11}^{+} \check{\ell}_{21}^{-} \frac{1}{\check{x}^{1}}
\end{aligned}
$$

Here we used $\check{V} \equiv \check{U}^{\frac{1}{3}}$. Algebraical consistency $\left(\check{\partial}_{1} \check{\partial}_{2}=q^{-1} \check{\partial}_{2} \check{\partial}_{1}\right)$ would however require an additional relation between the $L$-functionals and the coordinates. This requirement is fulfilled, if one expresses the operators $\check{\ell}_{12}^{+}$and $\check{\ell}_{21}^{-}$as follows:

$$
\begin{aligned}
& \check{\ell}_{12}^{+}=-q^{-2}\left(\check{\ell}_{22}^{+}-q^{2} \check{\ell}_{11}^{+} \check{V}^{2}\right) \check{x}^{2} \frac{1}{\check{x}^{1}} \\
& \check{\ell}_{21}^{-}=-q^{2}\left(\check{\ell}_{22}^{+}-q^{-2} \check{\ell}_{11}^{+} \check{V}^{-2}\right) \check{x}^{1} \frac{1}{\check{x}^{2}}
\end{aligned}
$$

\section{Remarks and Conclusions}

Of course, the whole gauge theory can be formulated by using directly differential forms [22]. As usual the covariant derivation and the gauge potential are then given by

$$
D \psi \equiv d \psi+A \psi, \quad A \equiv d \hat{x}^{i} A_{i}
$$


The gauge potential is transforming according to

$$
\delta A=d \Lambda+i[\Lambda, A]
$$

Which agrees with (43), if one takes into account that $\Lambda d \hat{x}^{i}=d \hat{x}^{j}\left(B_{j}{ }^{i} \Lambda\right)$. For the field strength one has

$$
i F=d A-i A A, \quad \delta F=i[\Lambda, F] .
$$

The torsion is related to the covariant derivation

$$
D e^{j} \equiv d e^{j}-i\left[A, e^{j}\right]
$$

of the vielbein field $e^{j}=d \hat{x}^{i} e_{i}{ }^{j}$.

However, if one works with $*$-products and has given a trace of the algebra (which we denote as $\int$ and where trace means in particular $\int f * g=\int g * f$ ), it seems to be more natural to use the field strength as defined in (47), since it transforms as $\delta F_{i j}=i\left[\Lambda * F_{i j}\right]$ and therefore for example

$$
\int \operatorname{Tr} F_{i j} * F^{i j}
$$

is invariant under gauge transformations ( $\mathrm{Tr}$ is a trace over the generators of the gauge group). In this way gauge invariant theories can be constructed [21].

In any case, this example shows, that if one wants to use some sort of "vielbein" in a noncommutative geometry, this vielbein has to transform nontrivially under gauge transformations.

Above, in section 4, we constructed the Seiberg-Witten map for the vielbein requiring that it reduces to 1 in the $h \rightarrow 1$ limit: $E^{(0)}{ }_{i}{ }^{j}=\delta_{i}{ }^{j}$. One could assume a $x$-dependent zeroth order term: $E^{(0)}{ }_{i}{ }^{j}=\varepsilon_{i}^{j}(x)$. In this case a Seiberg-Witten map also exists. E.g. a first order solution is:

$$
\begin{aligned}
& E^{(1)}{ }_{1}^{1}=-i \varepsilon_{1}^{1}\left(2 x^{1} a_{1}+x^{2} a_{2}\right)-i x^{1} x^{2}\left(a_{2} \partial_{1} \varepsilon_{1}^{1}-a_{1} \partial_{2} \varepsilon_{1}^{1}\right) \\
& E^{(1)}{ }^{2}=-i \varepsilon_{1}^{2}\left(x^{1} a_{1}+2 x^{2} a_{2}\right)-i x^{1} x^{2}\left(a_{2} \partial_{1} \varepsilon_{1}^{2}-a_{1} \partial_{2} \varepsilon_{1}^{2}\right)-i 2 x^{2} \varepsilon_{1}^{1} a_{1} \\
& E^{(1)}{ }^{1}=-i \varepsilon_{2}^{1}\left(2 x^{1} a_{1}+x^{2} a_{2}\right)-i x^{1} x^{2}\left(a_{2} \partial_{1} \varepsilon_{2}^{1}-a_{1} \partial_{2} \varepsilon_{2}^{1}\right) \\
& E^{(1)}{ }_{2}^{2}=-i \varepsilon_{2}^{2}\left(x^{1} a_{1}+2 x^{2} a_{2}\right)-i x^{1} x^{2}\left(a_{2} \partial_{1} \varepsilon_{2}^{2}-a_{1} \partial_{2} \varepsilon_{2}^{2}\right)-i 2 x^{2} \varepsilon_{2}^{1} a_{1}
\end{aligned}
$$

For the action of the matter fields one needs also fields $\bar{\psi}$ transforming according to $\delta \bar{\psi}=-i \bar{\psi} \Lambda$ :

$$
\delta \bar{\psi}=-i \bar{\psi} \Lambda, \quad \mathcal{D}_{i} \bar{\psi}=E_{i}{ }^{j}\left(\partial_{j} \bar{\psi}+i\left(B_{j}{ }^{k} \bar{\psi}\right) A_{k}\right) .
$$

A Seiberg-Witten map can be constructed along the above lines.

It would be interesting, to know if one can express the gauge field in terms of the vielbein (or the vielbein in terms of the gauge field). Inverting the SeibergWitten map for $A_{i}(70)$ and inserting $a_{i}\left[A_{j}\right]$ in the expression for the vielbein (69) this seems to be possible. Also the question arises, whether there are gauge and vielbein fields without torsion, apart from pure gauge fields. The 
Seiberg-Witten map does not give torsion free fields unless the commutative field strength $f_{12}$ vanishes:

$$
T_{12}^{1}=i h x^{1} f_{12}+\mathcal{O}\left(h^{2}\right), \quad T_{12}^{2}=2 i h x^{2} f_{12}+\mathcal{O}\left(h^{2}\right) .
$$

Furthermore, our results demonstrate, at least for special cases, that for algebras corresponding to a nonconstant Poisson-tensor $\theta^{i j}$ it is possible to construct covariant derivatives. Also some sort of Seiberg-Witten map exists. Having in mind general coordinate transformations, which clearly lead to nonconstant Poisson-structures, it would be interesting to know, if there exists a formulation which works for the generic case and uses derivatives. Or, if one has to go back to covariant coordinates, which always exist, but don't possess a nice commutative limit.

\section{Acknowledgements}

I would like to thank J. Wess for useful discussions, B. Zumino for comments on the manuscript and the Theoretical Physics Group of the Lawrence Berkeley National Laboratory for kind hospitality.

This work was supported by the Max-Planck-Society, Germany. This work was supported in part by the Director, Office of Science, Office of High Energy and Nuclear Physics, Division of High Energy Physics of the U.S. Department of Energy under Contract DE-AC03-76SF00098 and in part by the National Science Foundation under grant PHY-0098840.

\section{References}

[1] S.L. Woronowicz, Differential Calculus on Compact Matrix Pseudoproups (Quantum Groups), Commun. Math. Phys. 122 (1989) 125.

[2] J. Wess, B. Zumino, Covariant Differential Calculus on the Quantum Hyperplane, Nucl. Phys. Proc. Suppl. 18 B (1991) 302.

[3] N. Seiberg, E. Witten, String theory and noncommutative geometry, JHEP 9909 (1999) 032, hep-th/9908142.

[4] V.G. Drinfel'd, Quantum Groups, Proc. ICM, Berkeley 1987.

[5] S.L. Woronowicz, Compact Matrix Pseudogroups, Commun. Math. Phys. 111 (1987) 613.

[6] L.D. Faddeev, N.Y. Reshetikhin, L.A. Takhtajan, Quantization Of Lie Groups And Lie Algebras, Lengingrad Math. J. 1 (1990) 193.

[7] A.U. Klimyk, K. Schmüdgen, Quantum Groups and Their Representations, Springer Verlag, Berlin, 1998. 
[8] O. Ogievetsky, B. Zumino, Reality In The Differential Calculus On qEuclidean Spaces, Lett. Math. Phys. 25 (1992) 121, arXiv:hep-th/9205003.

[9] A. Lorek, W. Weich, J. Wess, Noncommutative Euclidean and Minkowski Structures, Z.Phys. C 76: 375-386, (1997).

[10] F. Bayen, M. Flato, C. Fronsdal, A. Lichnerowicz, D. Sternheimer, Deformation theory and quantization. I. Deformations of symplectic structures, Annals Phys. 111 (1978) 61.

[11] C. Bauer, H. Wachter, Operator Representations on Quantum Spaces, arXiv:math-ph/0201023.

[12] J. Madore, S. Schraml, P. Schupp, J. Wess, Gauge theory on noncommutative spaces, Eur. Phys. J. C16 (2000) 161, hep-th/0001203.

[13] H. Wachter, M. Wohlgenannt, *-Products on Quantum Spaces, arXiv:hepth/0103120.

[14] D. Brace, B.L. Cerchiai, A.F. Pasqua, U. Varadarajan, B. Zumino, A cohomological approach to the non-Abelian Seiberg-Witten map, JHEP 0106 (2001) 047, arXiv:hep-th/0105192.

[15] D. Brace, B.L. Cerchiai, B. Zumino, Non-Abelian gauge theories on noncommutative spaces, arXiv:hep-th/0107225.

[16] B. Jurčo, S. Schraml, P. Schupp, J. Wess, Enveloping algebra valued gauge transformations for non-Abelian gauge groups on noncommutative spaces, Eur. Phys. J. C17 (2000) 521, hep-th/0006246.

[17] M. Picariello, A. Quadri, S.P. Sorella, Chern-Simons in the SeibergWitten map for noncommutative Abelian gauge theories in $4 D$, arXiv:hepth/0110101.

[18] S.Schraml, Untersuchung nichtkommutativer Räume als Grundlage für physikalische Probleme, Dissertation, Ludwig-Maximilians-Universität München, Dec. 2000.

[19] B. Jurčo, P. Schupp and J. Wess, Nonabelian noncommutative gauge theory via noncommutative extra dimensions, Nucl. Phys. B 604 (2001) 148, [arXiv:hep-th/0102129].

[20] H. Steinacker, Integration on quantum Euclidean space and sphere in $N$ dimensions, LBL-37431.

[21] B. Jurčo, L. Möller, S. Schraml, P. Schupp, J. Wess, Construction of nonAbelian gauge theories on noncommutative spaces, Eur. Phys. J. C 21 (2001) 383, arXiv:hep-th/0104153.

[22] A. Dimakis, J. Madore, Differential calculi and linear connections, J. Math. Phys. 37 (1996) 4647. 